\title{
On Doppler Sensitivity of Transmission Waveforms for Active Sonar Detection
}

\author{
C. K. Sunith
}

\author{
Assistant Professor in Electronics, Model Engineering College, Thrikkakara, Kochi
}

\begin{abstract}
The development of an active sonar system requires information on the desired properties of the waveforms transmitted by the system. This article is based on MATLAB simulations and explores the Doppler sensitivity characteristics of various existing and proposed waveforms for underwater active sonar applications. As many as seven different waveforms were compared for Doppler sensitivities based on their matched filter responses.
\end{abstract}

Keywords: MATLAB, Active Sonar, Doppler Sensitivity, Matched Filter, Decimator, Beamformer, Normaliser.

\section{INTRODUCTION}

An active sonar system gathers information about a target by processing reflections or echo from the target. In an active sonar system, the received signal is processed with the help of matched filter arrangements, their output being the prime source of target information. Doppler sensitivity characteristics of the received signal depend also on the type of waveform used for transmission. Hence it becomes necessary to evaluate the Doppler sensitivity characteristics of a particular waveform before it is being put to use in active sonar applications.

\section{SIMULATION SETUP}

Simulations were carried out using MATLAB. The receiver block schematic used for simulations is given in Figure 1. As many as seven different pulsed waveforms were generated, each of a pulse width of 0.5 seconds $(0.5$ seconds on, 0.5 seconds off, total duration of one second). The types of waveforms generated were Pulsed Continuous Wave $(\mathrm{CW})$, Linear Frequency Modulated (LFM), Hyperbolic Frequency Modulated (HFM), Sinusoidal Frequency Modulated (SFM, for four different modulating frequencies) pulsed waveforms and pulsed waveforms based on frequency hopped, pseudo random noise sequences such as Costas Codes, Cox Comb and Quadratic Congruence Codes.

The waveforms after inverse beam forming and the addition of sea state (attenuation, random noise and reverberation) were applied to the receiver block. The receiver block essentially consists of a linear array of transducer elements spaced half wavelength apart. The front end processor conditions the array signal and converts it to digital signal for processing. The receiver will have to process the signal at a more convenient data rate. The signal is band pass filtered to improve SNR. Decimation process helps to sample the signal at a lesser data rate convenient to the receiver. The beamformer functions as a spatial filter to enhance detection capability, resolution and directional measurement of the plane wave signal and acts on the series of receiving elements which are called hydrophones. Receiving arrays are linear assemblies of hydrophones designed to increment signal to noise ratio and directionality. Array weights and shading coefficients are multiplied with the received signal to account for the attenuation suffered and improve directivity and to account for phase/time difference between different receiving elements depending on maximum response axis. Chebyshev polynomial coefficients were used as shading coefficients to suppress side lobe levels to as low as $23 \mathrm{~dB}$ below main lobe level.

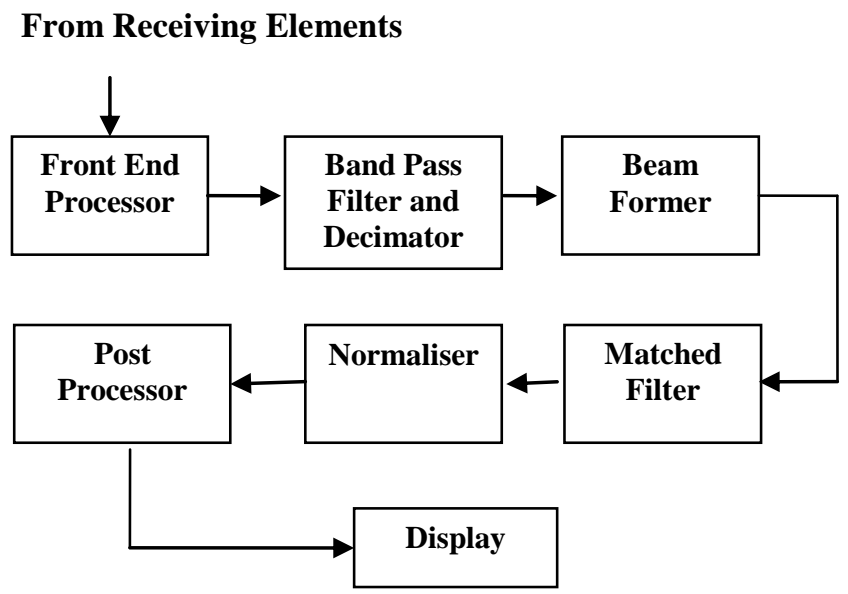

Fig 1 : Receiver Block Schematic

\section{RESULTS}

Doppler parameter (delta, $\delta$ ) is given by the expression

$$
\delta=2 \frac{V}{C}
$$

where $\mathrm{V}$ is the target velocity, $\mathrm{C}$ the sonic speed in water whose average value is equal to $1500 \mathrm{~m} / \mathrm{s}$. The expected value of $\delta$ was 0.02 . With $\delta$ varied from 0 to 0.04 , the peak 
Vol. 5, Issue 1, January 2017

amplitudes of matched filter output, after normalization with rms mean value of background noise were plotted along $\mathrm{Y}$ axis against values of delta along $\mathrm{X}$ axis.

A waveform is said to be Doppler sensitive if its matched filter output peaks only at the expected value of $\delta$ and show very low peak for the rest of the values of $\delta$. Simulations show that frequency hopped waveforms based on pseudo random noise sequences, such as Costas coded waveforms, Cox Comb and waveforms based on Quadratic Congruence Codes (QCC) do exhibit good Doppler sensitivity and tend to peak only at the expected value of $\delta=0.02$ (see Figure 2). Frequency modulated waveforms such as LFM and HFM tend to show low Doppler sensitivities with more than one peaks exceeding the threshold. SFM exhibited good Doppler sensitivity characteristic irrespective of the choice of modulating frequency. Pulsed CW waveform also showed good Doppler sensitivity during simulations.

\section{CONCLUSIONS}

Waveform design and selection have been potential candidates for continued research in the field of radar and sonar. Doppler sensitivity of waveform under study is a very important criterion that must be considered while selecting a waveform for use in active sonar applications. Implementation of a waveform that ideally suits all the required characteristics is a subject matter of continued research.
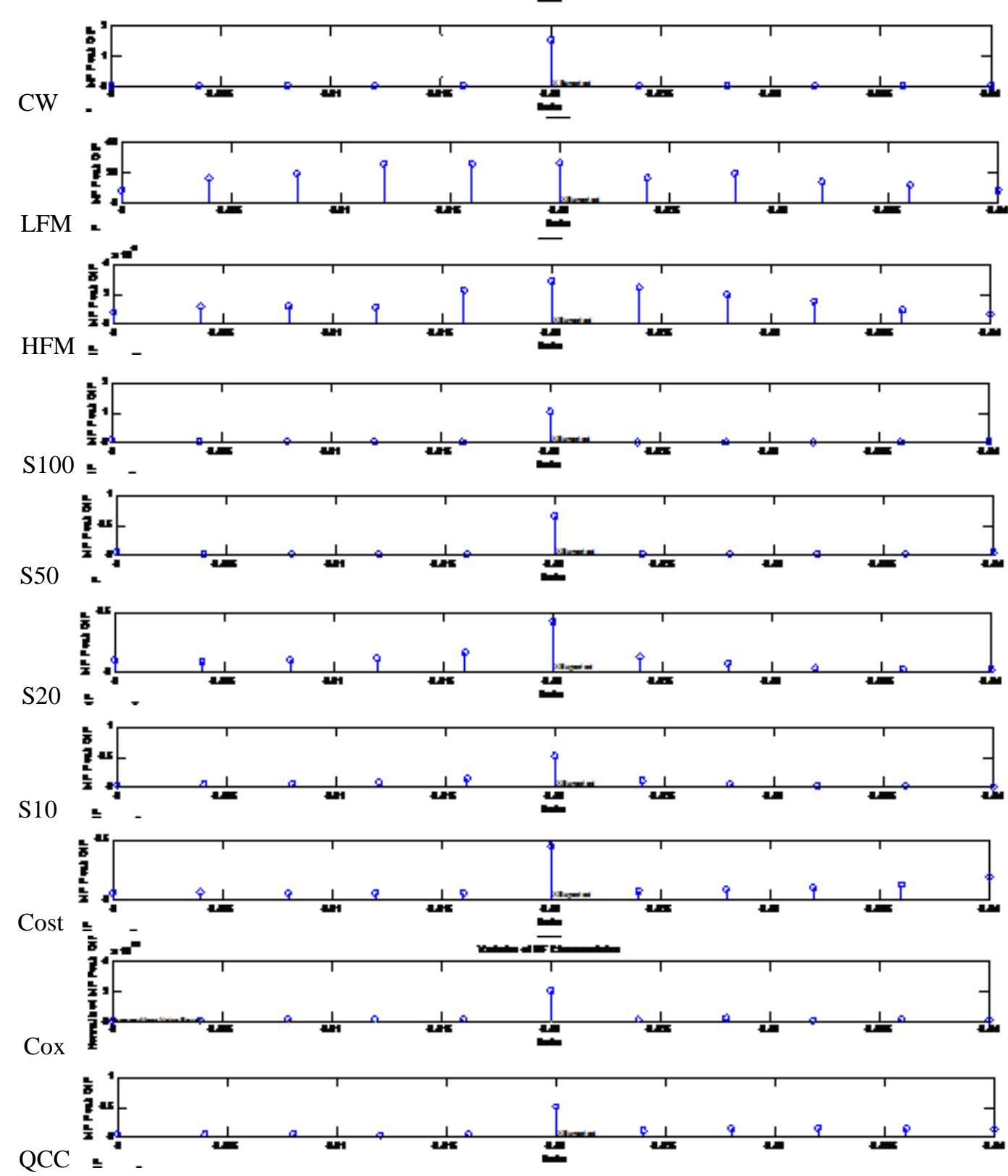

Fig 2 : Doppler sensitivity of waveforms $[\mathrm{S}<\mathrm{XXX}>/ \mathrm{S}<\mathrm{XX}>$ : SFM $<$ Modulating Frequency, Hz $>$, Cox $-\mathrm{Cox} \mathrm{Comb}]$ 


\section{IJIREEICE \\ International Journal of Innovative Research in Electrical, Electronics, Instrumentation and Control Engineering \\ ISO 3297:2007 Certified}

Vol. 5, Issue 1, January 2017

\section{REFERENCES}

[1]. Levanon, Nadav and Mozeson, N, "Radar Signals", IEEE Press , John Wiley and Sons.

[2]. Burdick, W S, "Underwater Acoustic System Analysis", Prentice Hall, Eaglewood Cliffs, NJ, 1984, ISBN 0-13-936716-0.

[3]. Winder, Alan A, "Sonar System Technology", IEEE Transactions on Sonics and Ultrasonics, Sept 1975.

[4]. Glisson, Black and Sage, "On Sonar Signal Analysis", IEEE Transactions on Aerospace and Electronic Systems, January 1970.

[5]. Costas, John P, "A Study of Class of Detection Waveforms having Nearly Ideal Range Doppler Ambiguity Properties", Proceedings of IEEE, August 1984.

[6]. Sun, Collins \& Xiao, University of Birmingham, "Target Tracking Using Wideband Cox Comb Waveforms for Human Computer Interaction".

[7]. Baldacci, Alberto \& Haralabus, Georgios, "Adaptive Normalisation of sonar data”, NURC-PR- 2006-13, August 2006.

[8]. Ricker, Dennis W, "The Doppler Sensitivity of Large TW Phase Modulated Waveforms", IEEE Transactions on Signal Processing, Vol. 40, No. 10, October 1993.

[9]. http://www.wikipedia.com

\section{BIOGRAPHY}

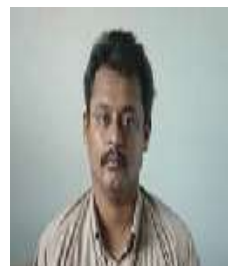

Mr. C K Sunith received his M Tech Degree from National Institute of Technology, Calicut. Presently he is working as Assistant Professor in Department of Electronics and Communication Engineering, Model Engineering College, Thrikkakara, Kochi - 21. He is an Associate Member of IETE, Associate Member of Institution of Engineers (India) and a Life Member of ISTE, Systems Society of India and Photonics Society of India. He has contributed papers and articles to national and regional journals and magazines. 